clear. We detected Ureaplasma urealyticum (Uu) and U parvum (Up) by quantitative PCR in the urine of men with and without NGU to show a possible association with urethritis.

Methods Urine samples from 158 male STD-clinic attendees with symptomatic NGU ( $>5 \mathrm{PMNL} / \mathrm{hpf}$ ) and 77 asymptomatic men without NGU ( $<5 \mathrm{PMNL} / \mathrm{hpf}$ ) were collected. The patient's age and number of partners within the previous 6 months were recorded. All samples were tested for Neisseria gonorrhoeae (Ng), Chlamydia trachomatis (Ct), Mycoplasma genitalium (Mg), Uu, Up, Trichomonas vaginalis (Tv), herpes simplex virus (HSV) 1 and 2, and adenovirus by real-time PCR.

Results Ct and Mg were found in 22 and $30 \%$ of NGU, respectively, and were associated with NGU ( $p<0.0001$ both). Three had dual Ct and $\mathrm{Mg}$ infection. Uu was detected in $13 \%$ of NGU cases and $12 \%$ of controls ( $p>0.99)$. The median Uu bacterial DNA load was higher in men with NGU than in men without (223 genome equivalents (geq) and 10 geq, respectively; $\mathrm{p}=0.002$ ). Using ROC-curve analysis to determine the optimal cut-off, patients with $>53$ geq were more likely to have urethritis $(p=0.02)$. In men with NGU of unknown aetiology, there was no difference in the rate of Uu detection when compared to controls $(p=0.26)$. The corresponding median $U u$ bacterial DNA load were significantly higher in this group than in controls $(p=0.01)$, and using a cut-off of $>53$ geq, men with NGU of unknown aetiology were more likely to harbour $U u(14 \%)$ than were men from the control group ( $1 \%),(p=0.005)$. Up was detected in $14 \%$ of NGU cases and $19 \%$ of controls $(p=0.34)$. There was no difference in the detection rate of Up or in the median Up bacterial load in any of the groups. HSV-1 was detected in $3 \%$ of cases and $1 \%$ of controls. HSV-2 was found in $2 \%$ of NGU cases. All urine samples were negative for adenovirus. Cases and controls had similar median number of partners within 6 months (2 partners) and age (28 and 29 years, respectively).

Conclusion The bacterial load of $U$ urealyticum in men with NGU and in men with NGU of unknown aetiology was higher than in men without NGU, and the presence of $>53$ geq of $U u$ was associated with urethritis in both groups. In accordance with other studies, U parvum was not associated with urethritis. (Preliminary results were presented at the ASM general meeting, San Diego 2010).

\section{P3-S7.15 DIAGNOSTIC RELIABILITY OF WET PREP MICROSCOPY FOR T VAGINALIS IN WOMEN VISITING A HIGH-VOLUME STD CLINIC}

doi:10.1136/sextrans-2011-050108.498

J Fitch, T Anderson, M Thrun, C Mettenbrink. Public Health Department, Denver, USA

Background For years, wet prep microscopy has been the cornerstone of testing for $T$ vaginalis due to the simplicity of the test and its low cost to perform. Though still recommended as a means of diagnosis for vaginal infections, its diagnostic sensitivity (typically $50 \%-70 \%$ ) falls short of other means of testing, including culture (approximately $80 \%$ sensitivity). This study compares the performance and costs of wet prep tests to culture in the hands of experienced laboratorians in a high volume STD clinic.

Methods The Denver Metro Health (STD) Clinic sees over 800 patients and performs over 100 wet prep tests every month. Between 1 January 2010 and 30 September 2010, a wet prep test and a $T$ vaginalis culture were performed on every female patient receiving a comprehensive exam, regardless of symptoms, as part of resistance testing for the STD Surveillance Network (SSuN). The results of the two tests were then compared.

Results Wet prep tests and $T$ vaginalis cultures were performed on 555 female patients. Of those, 51 were positive by wet prep and 62 were positive by culture see Abstract P3-S7.15 table 1. There were no wet prep false positives reported. The average material cost for each in-house wet prep was $\$ 0.32$ and the cost for each culture was $\$ 1.78$. In comparision, materials for PCR testing for $T$ vaginalis cost approximately $\$ 2$ per test. Staff time required to perform wet prep microscopy is less than either culture or PCR.

Conclusions The wet prep sensitivity rate of $82.2 \%$ when compared to culture (which, until recently has been the gold standard for trich testing) suggests that wet prep microscopy may be more useful than previously recognised in venues where clinicians and laboratorians gain experience by performing a large number of tests. Additionally, the costs, both in supply and staff expense, suggest that, in tight budgetary times, wet prep may continue to be a cornerstone of $T$ vaginalis testing, even with the availability of molecular amplification methods. These results, however, were limited to a single clinic during a single point in time. As next steps, wet prep testing might be compared to molecular amplification tests.

Abstract P3-S7.15 Table 1 Diagnostic reliability of wet prep microscopy for $T$ Vaginalis in women visiting a high-volume STD clinic

\begin{tabular}{lccc}
\hline & Wet prep & & \\
\cline { 2 - 3 } & Positive & Negative & Total \\
\hline Culture & & & \\
Positive & 51 & 11 & 62 \\
Negative & 0 & 493 & 493 \\
Total & 51 & 504 & 555 \\
\hline
\end{tabular}

\section{P3-S7.16 LACTOBACILLUS CRISPATUS COLONISATION REDUCES RISK OF BACTERIAL VAGINOSIS (BV) ACQUISITION}

doi:10.1136/sextrans-2011-050108.499

M Antonio, M Petrina, L Meyn, S Hillier. Magee-Womens Research Institute, University of Pittsburgh, Pittsburgh, USA

Background Longitudinal studies have found that vaginal colonisation by hydrogen peroxide-producing strains of lactobacilli is associated with a reduced risk of BV acquisition. Several Lactobacillus spp. including $L$ crispatus, $L$ jensenii, and $L$ gasseri can produce hydrogen peroxide, but no longitudinal studies to date have assessed whether all hydrogen peroxide producing species are equally protective. The objective of this study was to determine which species of Lactobacillus were associated with a reduced risk of BV acquisition.

Methods In this secondary analysis of women enrolled in a vaccine trial, 112 women aged 18-40 who had Gram stain Nugent scores $<6$ at study entry were followed bimonthly for up to 18 months for acquisition of BV. Vaginal and rectal swabs were collected at each visit for culture identification of lactobacilli. Species identification was performed by repetitive sequence PCR, and if necessary, 16S rRNA gene RFLP or sequencing. Cox proportional hazards models were used to assess the association between Lactobacillus colonisation at the prior visit and BV acquisition.

Results There were 40 BV acquisitions detected during 486 followup visits over 65.3 woman-years for an incidence of 61 per 100 woman-years. As shown in Abstract P3-S7.16 table 1, women colonised by $L$ crispatus were $53 \%$ less likely to acquire BV at a subsequent visit $(\mathrm{p}=0.023)$.

Conclusions $L$ crispatus was the only species protective against BV acquisition. Since neither $L$ jensenii nor $L$ gasseri reduced the risk of BV despite their capacity to produce hydrogen peroxide, other characteristics of $L$ crispatus may be more critical then peroxide production in protecting from BV acquisition. 